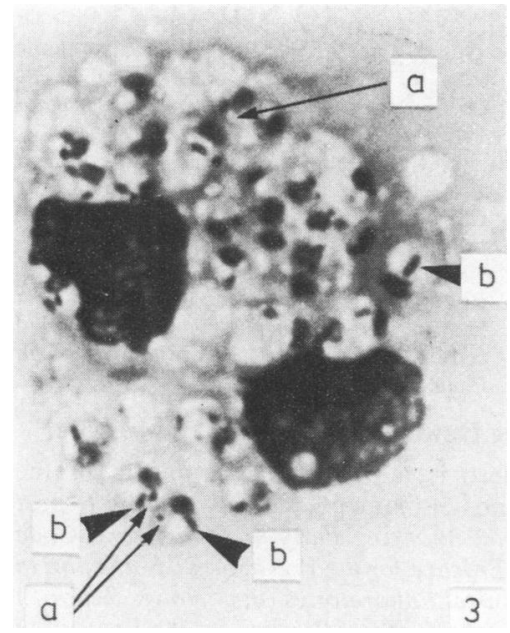

Fig. 3 A Leishman stained preparation showing a binucleated macrophage, from a hamster tissue smear, containing numerous amastigote forms of $L$ braziliensis braziliensis (LV63). The rod-shaped kinetoplasts-for example, $b$-and the much larger nuclei of the parasites are clearly distinguishable from the small rounded inclusions-for example, a-some of which appear brown under normal light but which are shown in Fig. 4 to be sharply refractile.

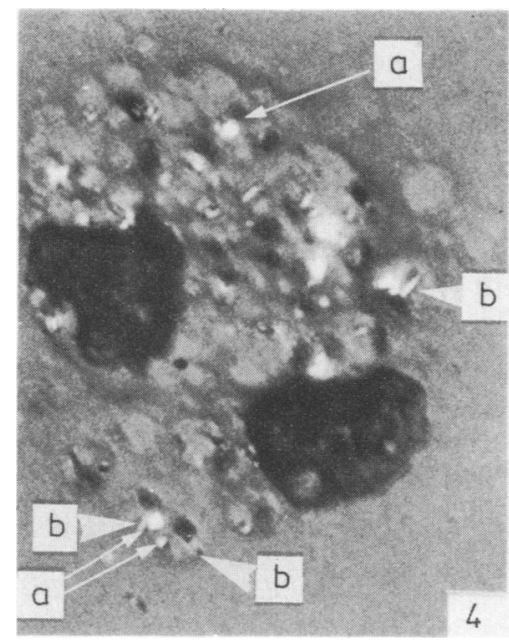

Fig. 4 The same field as in Fig. 3 under polarised light. The inclusions (a) show refractility, generally appearing larger and rounder than the rod-shaped kinetoplasts (b), but do not show a colour change on rotation-that is, they are not birefringent.
References

' Gardener PJ. Unidentified granule characterising an isolate of Leishmania braziliensis braziliensis. Trans $\mathrm{R}$ Soc Trop Med Hyg 1975;69:9.

${ }^{2}$ Scorza JV, Valera M, de Scorza C, Carnevali M, Moreno E, Lugo-Hernandez A. A new species of Leishmania parasite from the Venezuelan Andes region. Trans $R$ Soc Trop Med Hyg 1979:73:293.

\section{A selective agent for anaerobic cocci}

Anaerobic cocci often occur in combination with other organisms, notably $\mathrm{Bac}$ separation of anaerobic cocci from mixed cultures can be difficult and timeconsuming, especially as there are no satisfactory selective media for these organisms. Most of the selective media that have been described for anaerobes select either Gram-positive and Gram-negative anaerobes together or Gram-negative anaerobes alone. Wren, in his assessment of selective media for anaerobes ${ }^{2}$ found that a nalidixic acid-Tween medium gave the best recovery of anaerobic cocci from clinical samples-yet this medium also allowed good growth of most of the isolates of Gram-negative anaerobes. There is therefore a need for a selective medium that can select out Gram-positive anaerobes, especially anaerobic cocci, from mixed cultures that include Bacteroides spp. This letter describes in brief the use of bicozamycin $^{3}$ (bicyclomycin; CGP 354E; FR1881), an anti-diarrhoeal agent with specific activity against Gram-negative enteric pathogens, as a selective agent for anaerobic cocci. The results will be teroides spp, in clinical samples, ${ }^{1}$ yet the

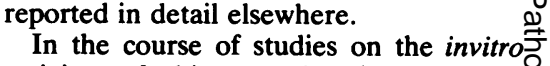
activity of bicozamycin (supplied by. Ciba-Geigy PLC) against anaerobes of clinical interest (Watt and Brown, to be published), we found that whereas all of the test anaerobic cocci were resistant to음 $256 \mathrm{mg} / \mathrm{l}$ of bicozamycin, almost all of the Bacteroides spp were inhibited at this con- $\triangle$ centration. We decided to assess the use of $10 \%$ horse blood agar containing $500 \mathrm{mg} / \mathrm{F}^{\infty}$ bicozamycin as a selective medium. The $\overrightarrow{0}$ bicozamycin was easily dissolved in distil- led water to give a stock solution of $50 \mathrm{~g} / \overrightarrow{\mathrm{w}}$ bicozamycin; $10 \mathrm{ml}$ of this solution was added to one litre Columbia blood agaro medium $^{4}$ while the agar was still molten tow give a final concentration of $500 \mathrm{mg} / \mathrm{l}$. The final surface $\mathrm{pH}^{4}$ of this medium was 7.1. We compared the surface growth, as asses- $O$ sed visually, of a range of clinical isolateso and reference strains on blood agar with and without bicozamycin. The results (Table) show that whereas all of the 87 anaerobic cocci strains (including three Veillonella spp) and 18 of 21 strains of clos- $\vec{\bullet}$ tridia grew well on the medium, only 3 of 58 strains of Gram-negative anaerobic bacilli grew on this medium and then onlyo with difficulty. Further studies have confirmed the ability of the medium to select out anaerobic cocci or clostridia from

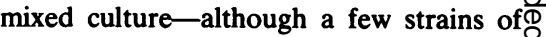
clostridia have failed to grow on the medium we have not encountered any윽 strains of anaerobic cocci that failed to grow. Indeed, quantitative tests have shown that the medium supports the growth of inocula of anaerobic cocci of $\leqslant \overline{0}$ $20 \mathrm{CFU} / \mathrm{ml}$.

The medium as described does allow

Growth of test anaerobes on media with and without bicozamycin

\begin{tabular}{|c|c|c|c|}
\hline \multirow[t]{2}{*}{ Test organism } & \multirow[t]{2}{*}{ No of strains } & \multicolumn{2}{|c|}{ No of strains showing growth on } \\
\hline & & $B A$ & $B B A$ \\
\hline $\begin{array}{l}B \text { fragilis } \\
B \text { distasonis } \\
B \text { thetaiotaomicron } \\
B \text { bivius } \\
\text { Other Bacteroides spp } \\
\text { Veillonella spp } \\
\text { C perfringens } \\
C \text { difficile } \\
\text { Other clostridia } \\
\text { Eubacterium spp } \\
\text { Pst anaerobius } \\
\text { Pc magnus } \\
\text { Pc asaccharolyticus } \\
\text { Other anaerobic cocci }\end{array}$ & $\begin{array}{r}24 \\
4 \\
13 \\
6 \\
11 \\
3 \\
9 \\
5 \\
7 \\
1 \\
31 \\
21 \\
12 \\
20\end{array}$ & $\begin{array}{r}24 \\
4 \\
13 \\
6 \\
11 \\
3 \\
9 \\
5 \\
7 \\
1 \\
31 \\
21 \\
12 \\
20\end{array}$ & $\begin{array}{l}0 \\
1^{*} \\
2^{*} \\
0 \\
0 \\
3 \\
8 \\
5 \\
5 \\
1 \\
31 \\
21 \\
12 \\
20\end{array}$ \\
\hline
\end{tabular}

BA = Blood agar; BBA = bicozamycin blood agar

* Very poor surface growth as assessed visually.

(1)


growth of a number of aerobic organisms. We have found that addition of neomycin to the medium (neomycin-bicozamycin blood agar, "NBBA") increases its selectivity and inhibits growth of most aerobic species, including many strains of Pseudomonas aeruginosa. Initially the final concentration of neomycin was $70 \mathrm{mg} / \mathrm{l}$ but we have found that this concentration in the medium makes NBBA inhibitory for occasional strains of anaerobic cocci and would suggest a lower concentration of neomycin (30-40 $\mathrm{mg} / \mathrm{l})$ to avoid this problem. At present, we are evaluating the use of this medium in the diagnostic laboratory and preliminary results are encouraging. We are also developing a nalidixic acidbicozamycin blood agar as an alternative selective medium for the isolation of anaerobic cocci from clinical examples.

Bicozamycin, used alone or in combination with agents such as neomycin allows good selective recovery of anaerobic cocci from mixed anaerobic cultures or from clinical material.

We thank Ciba-Geigy PLC for financial support.

B WATT FV BROWN

Bacteriology Laboratory, City Hospital, Greenbank Drive, Edinburgh EH10 5SB

\section{References}

${ }^{1}$ Holland JWE, Hill EO, Altemeier WA. Numbers and types of anaerobic bacteria isolated from clinical specimens since 1960. J Clin Microbiol 1977;5:20-5.

${ }^{2}$ Wren MWD. Multiple selective media for the isolation of anaerobic bacteria from clinical specimens. J Clin Pathol 1980;33:61-5.

${ }^{3}$ Nishida M, Yasuhiro M, Matsubara T. Bicyclomycin, a new antibiotic. III. In vitro and in vivo antimicrobial activity. $J$ Antibiot (Tokyo) 1972;25:582-93.

${ }^{4}$ Watt B, Jack EP. What are anaerobic cocci? J Med Microbiol 1977;10:461-8.

\section{Leukaemia/lymphoma cells in cerebro- spinal fuid}

The paper by Pearson et al in the December 1982 issue $^{1}$ is of interest in showing a further means of demonstrating tumour markers in cells harvested from the cerebrospinal fluid. Previously, Bradstock et $a l^{2}$ have demonstrated leukaemic cells in CSF using an anti-TdT antibody by indirect immunofluorescence. We have also recently been able to confirm the $B$ cell nature of a 'histiocytic' transformation occurring in the CSF in a case of M $\lambda$ B-CLL by the negative reaction with OKT3, 11, 4 and 8 combined with a positive reaction with OKIA and the presence of both IgM and $\lambda$ chains (shown by an indirect immunoperoxidase technique). We would like to suggest that while these methods are of importance in the positive diagnosis of malignant cells, they may also be put to valuable use in the exclusion of malignancy as the cause of symptomatology. We report one such case.

A farmer's ten-year-old daughter was diagnosed as having CALLA positive lymphoblastic leukaemia and was entered in the UKALL VI trial and received two years maintenance chemotherapy after remission induction. Cerebrospinal fluid was normal at diagnosis, during the CNS prophylaxis and at the completion of the therapy. Eleven months after stopping chemotherapy, she presented with acute symptoms suggestive of meningeal leukaemic relapse. Lumbar puncture showed her CSF to contain 175 leucocytes per microlitre, the vast majority of these being lymphocytes of atypical basophilic appearance, although not frankly lymphoblastic. Cytospin preparations of CSF were negative for CALLA ${ }^{3}$ and also TdT by indirect immunofluorescence. Appropriate positive and negative controls were included.

We feel this case shows the value of identifying the tumour markers at diagnosis, so that they may be used to identify or exclude the presence of malignant cells when appearing under suspicious circumstances at a later stage of the patient's illness. The value of using cytospin preparations is clear as the yield of cells from the patient's CSF would have been insufficient for live cell staining techniques. Although the patient received methotrexate at the time of the demonstration of the raised leucocyte count in her CSF, it is inconceivable that one injection of intrathecal methotrexate would control meningeal leukaemia for a period in excess of six months. We therefore conclude that the inability to demonstrate tumour cell markers in this case was a clear indication that the cells in the CSF were non-malignant and that the technique of examining cytocentrifuge CSF cell preparations using monoclonal antibodies is a viable addition to our investigative procedures.

$$
\begin{array}{r}
\text { HELEN MAGENNIS } \\
\text { GERALDINE MARKEY } \\
\text { HD ALEXANDER } \\
\text { TCM MORRIS } \\
\text { Department of Haematology, } \\
\text { Belfast City Hospital, } \\
\text { Lisburn Road, } \\
\text { Belfast BT9 7AD, } \\
\text { Northern Ireland }
\end{array}
$$

\section{References}

' Pearson J, Ilgren EB, Spriggs AI. Lymphon cells in cerebrospinal fluid confirmed by chromosome analysis $J$ Clin Path 1982;35:1307-11.

${ }^{2}$ Bradstock KF, Papageorgiou ES, Janossy G, al. Detection of leukaemic lymphoblasts CSF by immunofluorescence for termin F transferase. Lancet 1980;i:1144.

${ }^{3}$ Markey GM, Alexander HD, Morris TC\$ Robertson JH. A rapid method for identification of surface antigens on fixed cells using monoclonal antibodies. $J$ Clim Pathol 1982;35:1295-6.

The Howie report and the Howie code

There is a regrettable confusion in the minds of many people between the Repott of the Working Party to Formulate a Code of Practice for the Prevention of Infection in Clinical Laboratories (the "Howie Report' and the Code of Practice for the Preventio of Infection in Clinical Laboratories and Post-mortem Rooms (the "Howie Code").

The Report, which included the Code Practice, was submitted to the Chief ScF entific Officer of the Department of Healtit and Social Security in January 19780 Although an early draft had been "leaked" and was published in a trades union journg the Report has not been published by the Department. The reasons for this werf never made clear to members of the Worle ing Party which produced it, nor to the newspapers which supported publication. It may be relevant here to note that ong one of the five recommendations made $:$ 角 the Report (testing of equipment by the PHLS) has been implemented. For detais see Howie and Collins. ${ }^{1}$

The Code of Practice was publishe however, late in 1978, after the Birming ham smallpox incident but was not received in clinical laboratories until 1978 Unfortunately it contained several errors which would have been corrected had the proofs been read by members of the Working Party.

We ask, therefore, that Editors, authors laboratory workers and officials do not use the words "Howie Report" when they refer to the ("Howie") Code of Practice. JW HOWIE⿱ CH COLLINSt *34Redford Avenue Edinburgh EH13 OBE $\dagger$ The Ashes, Hadlow Kent TN11 OAS

\section{Reference}

' Howie JW, Collins CH. The Howie Code fo preventing infection in clinical laboratories尺 comments on some general criticisms and specific complaints. $\mathrm{Br} \quad \mathrm{Med}$ 1980;280:1071.

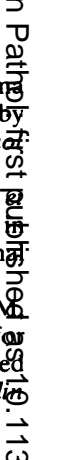

.

